\title{
Structural ordering at solid-liquid interfaces in Al-Sm system: a molecular- dynamics study
}

Yang Sun ${ }^{1,2}$, Feng Zhang ${ }^{2 \dagger}$, Zhuo Ye ${ }^{2}$, Zejun Ding ${ }^{1}$, Mikhail I. Mendelev ${ }^{2}$, Matthew J. Kramer ${ }^{2}$, Cai-Zhuang

$$
\text { Wang }^{2,3}, \text { Kai-Ming } \mathrm{Ho}^{1,2,3 *}
$$

${ }^{l}$ Hefei National Laboratory for Physical Sciences at the Microscale and Department of Physics, University of Science and Technology of China, Hefei, Anhui 230026, China

${ }^{2}$ Ames Laboratory, US Department of Energy, Ames, Iowa 50011, USA

${ }^{3}$ Department of Physics, Iowa State University, Ames, Iowa 50011, USA

Keywords: Solid-liquid interfaces; Structural ordering; Molecular dynamics simulations; Al-Sm.

\section{Abstract}

The structural ordering at solid-liquid interfaces far from equilibrium is studied with molecular dynamics simulations for the Al-Sm system. Using the van-Hove self-correlation function as the criterion to identify attachment/detachment events that occur at the interface, we are able to determine the time-dependent interface position, and characterize the detailed interfacial structure ordering surrounding the attached atoms. For the interface between an undercooled $\mathrm{Al}_{90} \mathrm{Sm}_{10}$ liquid and a metastable cubic structure, the solid induces the crystalline order of the cubic phase in the liquid layers, promoting the continuous growth of the crystal phase. When the same liquid is put in contact with f.c.c. Al, Sm from the liquid can still attach to the solid interface despite its insolubility in the Al lattice. Non-f.c.c. order is revealed surrounding the attached Sm atoms. We show that the local structure ordering at interface is highly correlated to solid packing and liquid ordering.

\section{Introduction}

Knowledge of solid-liquid interface is important to understand various phenomena, such as homogeneous/heterogeneous nucleation, melting, and wetting[1,2]. As the experimental studies on such interfaces are difficult and rare, atomistic simulations have guided a number of phenomenological understanding of solid-liquid interfaces [3-9]. Among the many unresolved puzzles, a fundamental topic is to understand the structural ordering at a solid-liquid interface [10-20]. This ordering can be characterized in

Authors to whom correspondence should be addressed:

† Email address: fzhang@ameslab.gov (Feng Zhang)

* Email address: kmh@ ameslab.gov (Kai-Ming Ho)

(C) 2016. This manuscript version is made available under the Elsevier user license http://www.elsevier.com/open-access/userlicense/1.0/ 
directions both perpendicular $[2,16,21]$ and lateral to the interface [22-24]. The lateral ordering, which is important in controlling the interfacial properties[25], has always been much more challenging to discern than its perpendicular counterpart, in both experiments and computer simulations, especially when the solid-liquid interface is out of thermodynamic equilibrium. In this work, we use dynamics-based parameters to characterize structural ordering in moving interfaces involved in phase transformations of a typical glass forming system: $\mathrm{Al}_{90} \mathrm{Sm}_{10}$. When $\mathrm{Al}_{90} \mathrm{Sm}_{10}$ glass is gradually heated up, it first shows a polymorphic transformation to a single metastable "big cube" phase (BCP) at $456 \mathrm{~K}[26]$. With molecular dynamics (MD) simulations, $\mathrm{BCP}$ can grow rapidly in $\mathrm{Al}_{90} \mathrm{Sm}_{10}$ undercooled liquid, which is consistent with experimental observation[27]. It remains puzzling why the metastable BCP is selected over thermodynamic stable phases such as fcc-Al. While our previous work approached this question from the structure and dynamics of bulk phases[27], here, we examine the structural difference at interfaces formed by undercooled $\mathrm{Al}_{90} \mathrm{Sm}_{10}$ liquid with BCP and fcc-Al. Based on the MD simulations, we focus on studying the impact of the composition and orientation of the solid phase on the interfacial order, as well as the correlation between ordering and migration of solid-liquid interfaces.

\section{Methods}

The MD simulations are performed with a semi-empirical Finnis-Sinclair (FS) potential to model the interatomic interactions for Al-Sm system [28], using the Large-scale Atomic/Molecular Massively Parallel Simulator (LAMMPS)[29]. The initial solid-liquid configurations are constructed by conjoining the equilibrium solid and liquid structures at their common cross-sections and applied periodic boundary conditions to all three dimensions (Please refer to the Supplementary Data for more simulation details). During the simulation, we allow the length of the box in the direction perpendicular to the interface to change, resulting in constant area, constant normal pressure: $N P_{x} A T$ ensemble, with $x$ being the perpendicular direction. All the solid-liquid samples are annealed under the ensemble at $800 \mathrm{~K}$ for 285 ns.

To distinguish solid-like and liquid-like atoms, we calculated the van Hove self-correlation function

$G_{\mathrm{s}}(r, \Delta t)$ for all Sm atoms in the simulations. $G_{\mathrm{s}}(r, \Delta t)$ is defined as $G_{s}(r, \Delta t)=\frac{1}{N}\left\langle\sum_{i=1}^{N} \delta\left(\vec{r}+\vec{r}_{i}(0)-\vec{r}_{i}(\Delta t)\right)\right\rangle$, where $\Delta t$ is chosen to be $1.2 \mathrm{~ns},\langle\cdot\rangle$ averages for all the time steps during the simulation and $\delta(\cdot)$ is the threedimensional dirac function. $G_{\mathrm{s}}(r, \Delta t)$ is used to characterize the atomic mobility during the time interval $\Delta t$.

\section{Results and discussion}


During the MD simulation, the $\mathrm{BCP}$ continues to grow, while the $\mathrm{Al}$ phase is partially melted for all three orientations (The initial and final snapshots are provided in the Supplementary Data Fig. S1). Since the interfaces are moving and have significant roughness during the non-equilibrium process, the snapshots from a fixed position can hardly show a clear interface structure, especially for the in-plane orders. Here, we characterize the interface position based on vastly different mobility of atoms in the solid and liquid regions. As can be seen in Fig. 1(a), the van Hove self-correlation function of $\mathrm{Sm}$ atoms in $\mathrm{BCP} / \mathrm{Al}_{90} \mathrm{Sm}_{10}$ is well separated into two major peaks, which describe the vibrational and diffusional motions in solid and liquid regions, respectively. In particular, it is interesting to note that a peak develops in the small-r region in all $\mathrm{Al} / \mathrm{Al}_{90} \mathrm{Sm}_{10}$ simulations. Since the solubility of $\mathrm{Sm}$ in the $\mathrm{Al}$ phase is essentially zero[30], these peaks can only be contributed by the $\mathrm{Sm}$ atoms attached to the $\mathrm{Al}$ interfaces from the liquid region. Given this drastically different mobility of Sm atoms in solid and liquid regions, one expects a sudden decrease of the mobility when the Sm atom attaches to a lattice site at the solid interface from the liquid region. On the other hand, if the Sm atom detaches from the solid interface, its mobility should significantly increase. In Fig. 1(b) and (c), we trace the displacement of typical $\mathrm{Sm}$ atoms duing the previous $\Delta t=1.2 n s$ as $D(t)=$ $|\vec{r}(t)-\vec{r}(t-\Delta t)|$, in $\mathrm{BCP} / \mathrm{Al}_{90} \mathrm{Sm}_{10}$ and $\mathrm{Al}(100) / \mathrm{Al}_{90} \mathrm{Sm}_{10}$, respectively. In both cases, one can identify several sudden jumps associated with the attachment or detachment event, regardless whether the solid phase grows (BCP) or melts (Al). In particular, Sm can be solid-like for 5-10 ns after attachment at the $\mathrm{Al} / \mathrm{Al}_{90} \mathrm{Sm}_{10}$ interfaces (shown in the insert of Fig. 1(c)), although it is insoluble in the Al lattice. We counted the life time of attached Sm atoms in each interface and showed the distributions in Fig. 1(d). One can see a number of Sm atoms staying at $\mathrm{Al} / \mathrm{Al}_{90} \mathrm{Sm}_{10}$ interfaces without any diffusion for more than $5 \mathrm{~ns}$. Here, a cut-off value of 1.2 $\AA$, which is the first minimum in $G_{\mathrm{s}}(r, \Delta t)$, is chosen as the criteria to distinguish liquid-like and solid-like behaviors. 

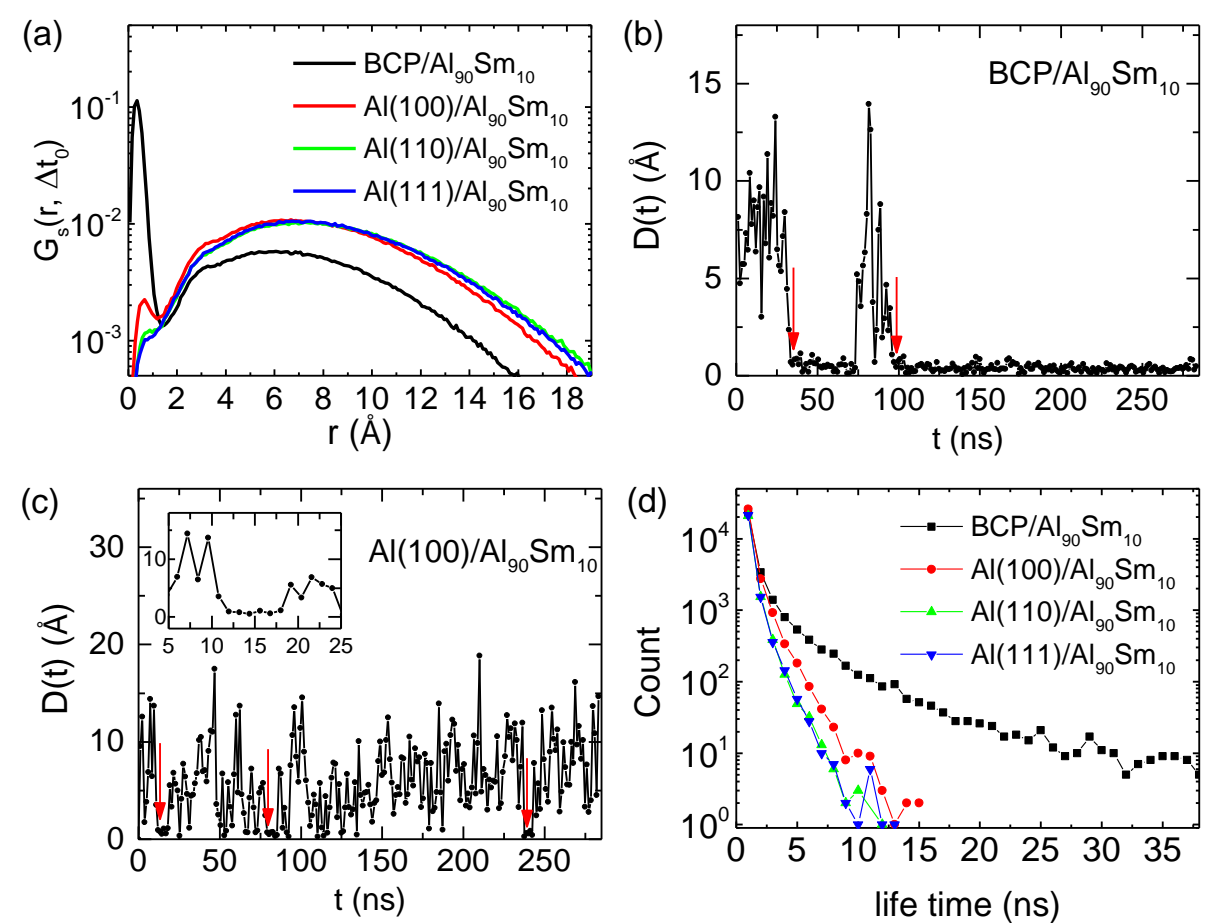

Fig. 1 (a) The van Hove self-correlation function for all Sm atoms in the simulations. (b) and (c) Typical histories of $\mathrm{Sm}$ atom displacement in $\mathrm{BCP} / \mathrm{Al}_{90} \mathrm{Sm}_{10}$ and $\mathrm{Al}(100) / \mathrm{Al}_{90} \mathrm{Sm}_{10}$ simulations, respectively. The arrow indicates a sharp drop corresponding to the atom attachment. The insert in (c) zooms in the first attachment event to show the solid-like life time. (d) The distribution of solid-like life time after the attachment.

The position where the attachment event occurs should reliably represent the time-dependent solidliquid interface position. Consequently, the interfacial structure ordering can be revealed by studying the local structures surrounding the attached atoms. For the purpose of achieving a statistically sound analysis, we superpose the local atomic distribution surrounding all the attachment events that occur on symmetrically equilavent crystal sites. Then, each atom is represented by a gaussian function to convert atom distribution to density distribution as: $\rho(\vec{r})=\sum_{i}\left(\frac{\alpha}{\pi}\right)^{3 / 2} e^{-\alpha\left(\vec{r}-\vec{r}_{i}\right)^{2}}$, where $\vec{r}_{i}$ is the position vector of atom $i$.

In Fig. 2(a)-(d), we first project the density distribution of local interface to one-dimenssional profile as a function of $x$, the direction normal to the interface plane. The dash line at $x=0 \AA$ indicates the position of $\mathrm{Sm}$ atom attachment. The density profile on the left of the attachment position shows peaks corresponding to the crystal planes, with different spacing and widths corresonding to the different geometry. When $x$ approaches to the interface, the density peaks decrease in amplitude and increase in width due to the 
increasing of Debye-Wallor factors [22]. These density peaks continue attenuating oscillation in liquid region for more than $10 \AA$. We also show the density profiles at $t_{0}-\Delta t$ (1.2 ns before attachment), assuming the attachment occurs at $t_{0}$. As can be seen in Fig. 2, all the density profiles at $t_{0}-\Delta t$ and $t_{0}$ almost overlap, indicating that the interface position almost does not change during this short time interval.

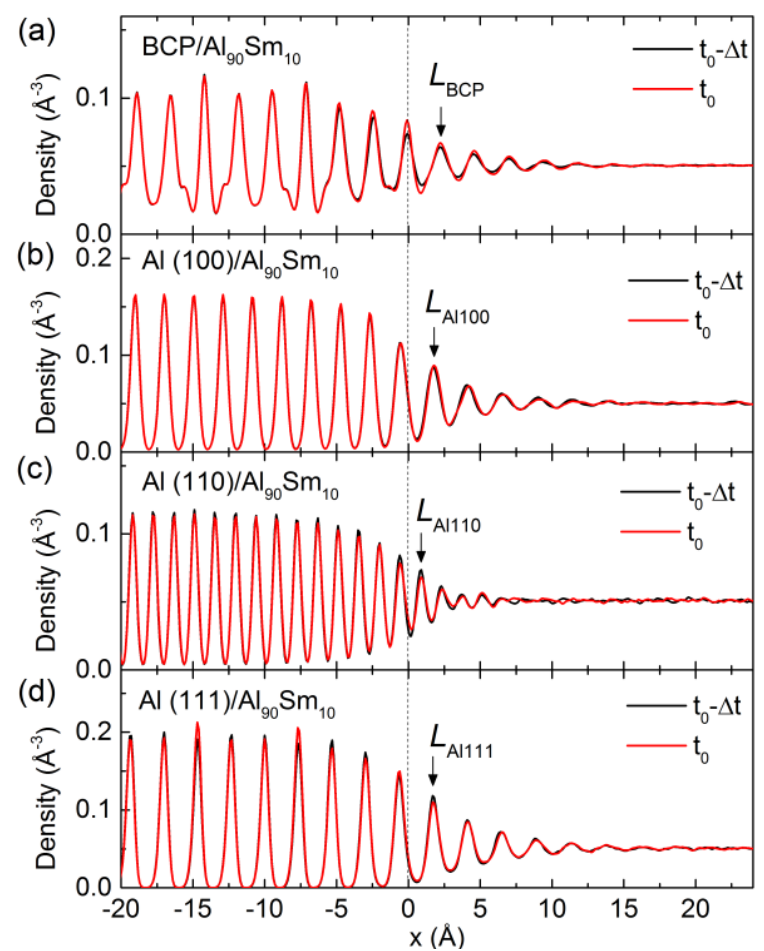

Fig. 2 The density profiles near the attachment position for the interface between (a) BCP, (b) $\mathrm{Al}$ (100), (c) $\mathrm{Al}$ (110) and (d) $\mathrm{Al}$ (111) contacting with $\mathrm{Al}_{90} \mathrm{Sm}_{10}$ liquid. The black curves show the density profile $1.2 \mathrm{~ns}$ before the attachment, while red curves show the one after attachment. The dashed lines indicate the attachment positions. The interfacial layers induced in the liquid region are labelled with $L_{\mathrm{BCP}}, L_{\mathrm{Al} 100}, L_{\mathrm{Al} 110}$ and $L_{\mathrm{Al111}}$, respectively.

The density profile allows us to clearly distinguish the layering effect from solid to liquid region. To visualize the lateral orders at the interface, we extract the in-plane density distributions at the layers labelled in Fig. 2 with a thickness of $0.5 \AA$, which is smaller than the half width of the density peaks. Figure 3 shows the lateral orders at $t_{0}-\Delta t$ and $t_{0}$ for each interfacial layer. The projection of the attachment position is centered at $(y=0, z=0)$ on each plane. As shown in the left pannel of the figures, all the interfacial layers have already shown inhomogenious atom distributions prior to the $\mathrm{Sm}$ attachment. The pattern of the atom distribution is correlated to the order of the corresponding solid phase. After attachment, the atom distribution in $L_{\mathrm{BCP}}$ keeps 
the same motif, and the order intensity gets enhanced as shown in the right pannel of Fig. 3(e). However, in the right pannel of Fig. 3(f)-(h), the ordering surronding the center of the $L_{\mathrm{Al100}}, L_{\mathrm{Al110}}$ and $L_{\mathrm{Al111}}$ is changed dramatically due to Sm attachment. In $L_{\mathrm{Al1} 100}$, the packing motif changes from cubic symmetry to a distorted hexagon, indicating the previous f.c.c order is broken and new motifs rebuilt. Also, in $L_{\mathrm{Al} 110}$ and $L_{\mathrm{Al} 111}$, the original fcc orders transform to a diamond and hexagnol motifs, respectively. Recent study[31] indicates that certain short-range order surronding Sm atoms with larger coordination numbers ( 16) shows relatively low energy in both AlSm liquid and crystal phases. Thus, when Sm attaches to the Al interface, it destroys the preexisting f.c.c. order, in order to assemble the energetically preferred orders. Such new order in turn traps the attached Sm atom, so that it can stay at interface for a few nanoseconds even it can not penetrate into the fcc lattice. However, these orders can only be formed locally and do not commeasurate with f.c.c. periodicity, thus the $\mathrm{Sm}$ atoms will eventually detach from the $\mathrm{Al}$ interface. The different ordering in the interfacial layers caused by the Sm attachment is consistent with our observation in the MD simulations that only BCP can continuously grow while the $\mathrm{Al}$ phase melts in A190Sm10 undercooled liquid.

(a)

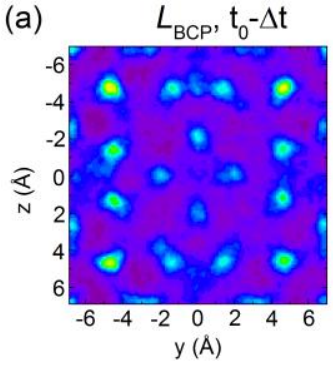

(c)

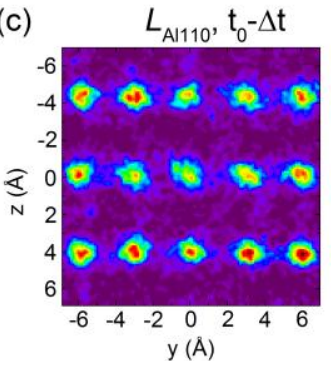

$L_{\mathrm{BCP}}, \mathrm{t}_{0}$
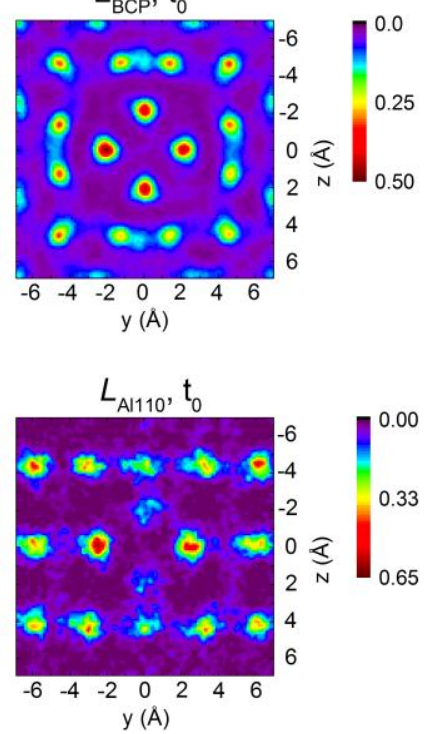

(b)

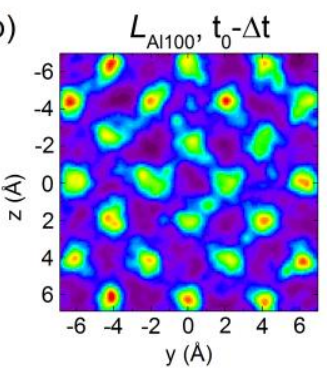

(d)

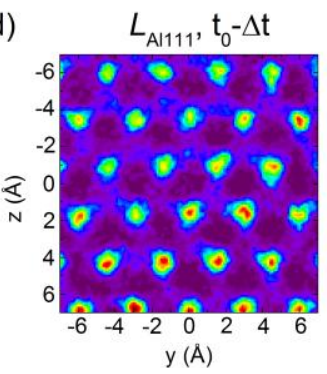

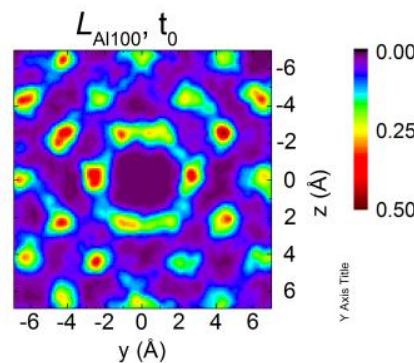

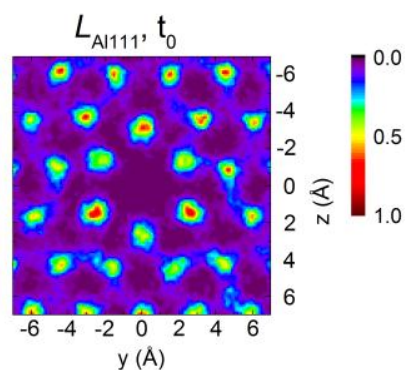

Fig. 3 (a)-(d) show the changes of lateral orders due to $\mathrm{Sm}$ atom attachment in the interfacial layers of $\mathrm{BCP} / \mathrm{Al}_{90} \mathrm{Sm}_{10}, \mathrm{Al}(100) / \mathrm{Al}_{90} \mathrm{Sm}_{10}, \mathrm{Al}(110) / \mathrm{Al}_{90} \mathrm{Sm}_{10}$ and $\mathrm{Al}(111) / \mathrm{Al}_{90} \mathrm{Sm}_{10}$, respectively. The left panel in each figure is $1.2 \mathrm{~ns}$ before $\mathrm{Sm}$ attachment, while right panel is right after $\mathrm{Sm}$ attachment.

\section{Conclusions}


Using MD simulations, we have carried out the studies about local structural ordering at both growing and melting solid-liquid interface for Al-Sm system. By employing the van-Hove self-correlation functions to identify attachment/detachment events occurred at the interface, we are able to determine the time-dependent interface position, and characterize the detailed interfacial structure ordering surrounding the attached atoms. We show that crystal will always induce structural ordering into contacting liquid regardless whether the crystal phase is growing or melting in the liquid. In the lateral direction, the energetically favored orders can form rapidly surrounding the attached atoms; while the density profile in the longitudinal direction responses much slower to the attachment. For the growing interface, the atom attachment can enhance the pre-existing order in the liquid. While in the melting interface, atom attachment can break the crystal orders and form new ones that are incompatible with the original packing motifs. Such mechanism gives rise to frequently attachment behavior even when the atom has no solubility in the solid phase.

\section{Acknowledgements}

Work at Ames Laboratory was supported by the US Department of Energy, Basic Energy Sciences, Division of Materials Science and Engineering, under Contract No. DE-AC02-07CH11358, including a grant of computer time at the National Energy Research Supercomputing Center (NERSC) in Berkeley, CA. Z.J.D. acknowledges support from the National Natural Science Foundation of China (No. 11274288) and the National Basic Research Program of China (No. 2011CB932801 and No. 2012CB933702).

\section{References}

[1] Y. Mishin, M. Asta, J. Li, Atomistic modeling of interfaces and their impact on microstructure and properties, Acta Mater. 58 (2010) 1117-1151.

[2] W.D. Kaplan, Y. Kauffmann, Structural Order in Liquids Induced by Interfaces with Crystals, Annu. Rev. Mater. Res. 36 (2006) 1-48.

[3] R.L. Davidchack, B.B. Laird, Weighted-density approximation for general nonuniform fluid mixtures, Phys. Rev. E. 60 (1999) 3417-3420.

[4] R.L. Davidchack, B.B. Laird, Simulation of the hard-sphere crystal-melt interface, J. Chem. Phys. 108 (1998) 9452.

[5] M. Amini, B.B. Laird, Crystal-melt interfacial free energy of binary hard spheres from capillary fluctuations, Phys. Rev. B. 78 (2008) 144112.

[6] C.A. Becker, M. Asta, J.J. Hoyt, S.M. Foiles, Equilibrium adsorption at crystal-melt interfaces in Lennard-Jones alloys., J. Chem. Phys. 124 (2006) 164708.

[7] C.A. Becker, D. Olmsted, M. Asta, J.J. Hoyt, S.M. Foiles, Atomistic underpinnings for orientation selection in alloy dendritic growth., Phys. Rev. Lett. 98 (2007) 125701.

[8] D.Y. Sun, M.I. Mendelev, C.A. Becker, K. Kudin, T. Haxhimali, M. Asta, et al., Crystal-melt interfacial free energies in hcp metals: A molecular dynamics study of Mg, Phys. Rev. B. 73 (2006) 024116.

[9] J.M. How, Direct observation of order in the liquid at a solid-liquid interface by high-resolution transmission electron microscopy, Philos. Mag. A. 74 (1996) 761-775.

[10] D. Kaminski, P. Poodt, E. Aret, N. Radenovic, E. Vlieg, Observation of a liquid phase with an 
orthorhombic orientational order., Phys. Rev. Lett. 96 (2006) 056102.

[11] S.H. Oh, Y. Kauffmann, C. Scheu, W.D. Kaplan, M. Rühle, Ordered liquid aluminum at the interface with sapphire, Science 310 (2005) 661-663.

[12] T.U. Schülli, R. Daudin, G. Renaud, A. Vaysset, O. Geaymond, A. Pasturel, Substrate-enhanced supercooling in AuSi eutectic droplets, Nature. 464 (2010) 1174-1177.

[13] S.E. Donnelly, R.C. Birtcher, C.W. Allen, I. Morrison, K. Furuya, M. Song, et al., Ordering in a fluid inert gas confined by flat surfaces., Science. 296 (2002) 507-10.

[14] H. Reichert, O. Klein, H. Dosch, M. Denk, V. Honkimäki, T. Lippmann, et al., Observation of fivefold local symmetry in liquid lead, Nature. 408 (2000) 839-841.

[15] S. Tsukimoto, S. Arai, M. Konno, T. Kamino, K. Sasaki, H. Saka, In situ high resolution electron microscopy/electron energy loss spectroscopy observation of wetting of a Si surface by molten $\mathrm{Al}$, J. Microsc. 203 (2001) 17-21.

[16] F. Spaepen, A structural model for the solid-liquid interface in monatomic systems, Acta Metall. 23 (1975) 729-743.

[17] J.Q. Broughton, A. Bonissent, F.F. Abraham, The fcc (111) and (100) crystal-melt interfaces: a comparison by molecular dynamics simulation, J. Chem. Phys. 74 (1981) 4029-4039.

[18] W.A. Curtin, Density-functional theory of the solid-liquid interface, Phys. Rev. Lett. 59 (1987) 12281231.

[19] B.J. Jesson, P.A. Madden, Structure and dynamics at the aluminum solid-liquid interface: An ab initio simulation, J. Chem. Phys. 113 (2000) 5935-5946.

[20] W.J. Huisman, J.F. Peters, M.J. Zwanenburg, S.A. de Vries, T.E. Derry, D. Abernathy, et al., Layering of a liquid metal in contact with a hard wall, 390 (1997) 379-381.

[21] C. Tang, P. Harrowell, Anomalously slow crystal growth of the glass-forming alloy CuZr, Nat Mater. 12 (2013) 507-511.

[22] Y. Yang, D.L. Olmsted, M. Asta, B.B. Laird, Atomistic characterization of the chemically heterogeneous Al-Pb solid-liquid interface, Acta Mater. 60 (2012) 4960-4971.

[23] D. Buta, M. Asta, J.J. Hoyt, Atomistic simulation study of the structure and dynamics of a faceted crystal-melt interface., Phys. Rev. E. Stat. Nonlin. Soft Matter Phys. 78 (2008) 031605.

[24] M. Guerdane, H. Teichler, B. Nestler, Local Atomic Order in the Melt and Solid-Liquid Interface Effect on the Growth Kinetics in a Metallic Alloy Model, Phys. Rev. Lett. 110 (2013).

[25] A.L. Greer, Liquid metals: Supercool order, Nat Mater. 5 (2006) 13-14.

[26] Y.E. Kalay, L.S. Chumbley, I.E. Anderson, Crystallization behavior in a highly driven marginal glass forming alloy, J. Non. Cryst. Solids. 354 (2008) 3040-3048.

[27] Z. Ye, F. Zhang, Y. Sun, M.C. Nguyen, M.I. Mendelev, R.T. Ott, et al., Unconventional phase selection in high-driven systems: A complex metastable structure prevails over simple stable phases, arXiv:1502.00023 (2015). http://arxiv.org/abs/1502.00023

[28] M.I. Mendelev, F. Zhang, Z. Ye, Y. Sun, M.C. Nguyen, S.R. Wilson, et al., Development of interatomic potentials appropriate for simulation of devitrification of Al90Sm10 alloy, Model. Simul. Mater. Sci. Eng. 23 (2015) 45013.

[29] S. Plimpton, Fast Parallel Algorithms for Short-Range Molecular Dynamics, J. Comput. Phys. 117 (1995) 1-19.

[30] S.H. Zhou, R.E. Napolitano, The Stability of Al11Sm3 (Al4Sm) Phases in the Al-Sm Binary System, Metall. Mater. Trans. A. 38 (2007) 1145-1151.

[31] Y. Sun, F. Zhang, Z. Ye, Y. Zhang, X. Fang, Z. Ding, C.-Z. Wang, et al., Crystal "Genes" in Metallic Liquids, Sci. Rep. (2016), in press. 